ЭПИДЕМИОЛОГИЯ ХРОНИЧЕСКОЙ БОЛЕЗНИ ПОЧЕК В РОССИЙСКОЙ ФЕДЕРАЦИИ ПО ДАННЫМ ФЕДЕРАЛЬНОГО РЕГИСТРА ВЗРОСЛЫХ ПАЦИЕНТОВ С САХАРНЫМ ДИАБЕТОМ (2013-2016 ГГ.)

( ) М.Ш. Шамхалова, О.К. Викулова*, А.В. Железнякова, М.А. Исаков, М.В. Шестакова, И.И. Дедов

ФГБУ Национальный медицинский исследовательский центр эндокринологии Минздрава России, Москва

ОБОСНОВАНИЕ. Хроническая болезнь почек (ХБП) - одно из наиболее тяжелых осложнений сахарного диабета, что определяет актуальность изучения эпидемиологических характеристик данной патологии.

ЦЕЛЬ. Оценить эпидемиологические характеристики развития ХБП у взрослых пациентов с СД 1 типа (СД1) и СД 2 типа (СД2) в РФ за период 2013-2016 гг.

МЕТОДЫ. Объектом исследования является база данных Федерального регистра СД - 81 региона РФ, включенных в систему онлайн-регистра. Оценивались показатели ХБП на 10000 взрослых больных СД (>18 лет).

РЕЗУЛЬтАТЫ. В 2016 г. частота регистрации ХБП составила: СД1 - 23\%, СД2 - 6,9\%, с выраженными межрегиональными различиями от 1,5\% до 49,9\% и от 0,6\% до 23,5\% соответственно при СД1 и СД2. Распространенность ХБП в динамике $2013 \rightarrow 2016$ гг. составила при СД1: 2171,4 $\rightarrow 2303,0 / 10$ 000, СД2: 512,8 $\rightarrow 687,2 / 10000$ взрослых. Частота регистрации новых случаев ХБП возросла в 2 раза при СД1 (215,5 против 104,2/10 000 взрослых), в 3,7 раза - при СД2 (190,4 против 51,8/10 000 взрослых). Анализ распределения по стадиям указывает на улучшение диагностики осложнения. В структуре ХБП выявлено увеличение доли пациентов с низким и умеренным риском сердечно-сосудистых событий и терминальной почечной недостаточности по критериям KDIGO (начальные стадии ХБП С1-2, A1-2): СД1 12,0\% $\rightarrow 46,8 \%$; СД2 $10,0 \% \rightarrow 50,4 \%$. Доля пациентов с очень высоким риском (стадии С-5, А2-3) уменьшилась: СД1 13,4\% $\rightarrow 6,7 \%$, СД2 $11,3 \rightarrow 4,4 \%$. Установлена зависимость распространенности ХБП от длительности диагноза СД. При СД1 <5 лет ХБП развивалась у 5,1\% пациентов, при СД1 <30 лет - у 48,0\%; СД2: 3,5\% и 20,3\% соответственно. Средний возраст дебюта ХБП у лиц с СД1 увеличился на 4,3 года $(36,1 \rightarrow 40,4)$ и 2,4 года при СД2 $(64,4 \rightarrow 66,8$ лет), длительность СД до момента диагностики ХБП увеличилась: СД1 - 11,8 $\rightarrow 14,2$ лет, СД 2 - 7,6 $\rightarrow 8,2$ лет.

ЗАКЛЮЧЕНИЕ. При общем увеличении распространенности ХБП в РФ в динамике 2013-2016 гг. отмечается улучшение качества диагностики осложнения на более ранних стадиях, в более позднем возрасте и при большей длительности СД. Успехи в ведении пациентов с СД в последние годы не снижают риск ХБП, но дают отсрочку в ее развитии. Выраженные межрегиональные различия в частоте регистрации ХБП в регистре указывают на проблемы диагностики ХБП в ряде регионов, где не выполняется стандарт обследования пациентов с СД с обязательной оценкой скорости клубочковой фильтрации (СКФ) и альбуминурии не реже 1 раза в год.

КЛЮЧЕВЫЕ СЛОВА: сахарный диабет; Федеральный регистр сахарного диабета; хроническая болезнь почек; риск сердечно-сосудистых событий

\title{
TRENDS IN THE EPIDEMIOLOGY OF CHRONIC KIDNEY DISEASE IN RUSSIAN FEDERATION ACCORDING TO THE FEDERAL DIABETES REGISTER (2013-2016)
}

(c) Minara S. Shamkhalova, Olga K. Vikulova*, Anna V. Zheleznyakova, Michail A. Isakov, Marina V. Shestakova, Ivan I. Dedov

Endocrinology Research Centre, Moscow, Russia

BACKGROUND: Chronic kidney disease (CKD) is one of the most severe complications of diabetes mellitus (DM), this determines the importance of the study of epidemiological characteristics of the disease.

AIMS: To assess the epidemiological characteristics of CKD in adult DM patients with type 1 (T1), 2 (T2) in Russian Federation in 2013-16.

METHODS: We have used the database of the Russian Federal Diabetes register, 81st regions included in online register. Indicators were estimated per 10,000 adult DM patients (>18years).

RESULTS: In 2016, the CKD frequency registration was T1 23\%, T2 6.9\% with marked interregional differences $1.5-49.9 \%$, $0.6-23.5 \%$, respectively. The CKD prevalence in dynamics $2013 \rightarrow 2016$ was $2171.4 \rightarrow 2303.0$ in T1 and 512 . $\rightarrow 687.2$ in T2. The incidence of new CKD cases increased 2 times in T1 (215.5 vs 104.2), and 3.7 times in T2 (190.4 vs 51.8). The analysis of distribution by CKD stages by KDIGO indicates the increase in the proportion of patients with low and moderate cardiovascular risk and end stage renal disease (ESRD) (with the initial stages of CKD, C1/2 A1) - 12.0 $\rightarrow 46.8 \%$ in T1; $10.0 \rightarrow 50.4 \%$ in T2. 
The proportion of patients with a very high risk (stages C4/5 C3aA3 and C3bA2-3) progressively decreases: $13.4 \rightarrow 6.7 \%$ in $\mathrm{T} 1,11.3 \rightarrow 4.4 \%$ in T2. We observed relation between the CKD prevalence and DM duration. CKD develops in $5.1 \%$ patients if T1 $<5$ years and in 48.0\% if T1 >30years; in T2 3.5\% and 20.3\%, respectively. The average age of CKD onset in T1 increased for $4,3 \mathrm{yr}(36,1 \rightarrow 40,2)$, in T2 for $2,4 \mathrm{yr}(64,4 \rightarrow 66,8)$, DM duration until CKD development increased in T1 $11.8 \rightarrow 14.2 \mathrm{yr}$, in T2 $7.6 \rightarrow 8.2 \mathrm{yr}$.

CONCLUSIONS: There is a significant improvement in the quality of CKD diagnostics at the earlier stages, older age and a longer DM duration before CKD onset in both types while we observed the increasing trends in CKD prevalence in Russian Federation in the dynamics of 2013-2016. Advances in the management of patients with DM in recent years do not reduce the risk of CKD, but give us a delay in its development. The marked interregional differences frequency of registration of CKD might indicate some remaining problems in verification in a number of regions where the standard for mandatory assessment of albuminuria and glomerular filtration rate not implemented.

KEYWORDS: diabetes mellitus (DM); the register of diabetes mellitus; chronic kidney disease (CKD); cardiovascular risk

\section{ОБОСНОВАНИЕ}

Хроническая болезнь почек (ХБП) у больных сахарным диабетом (СД) остается ведущим микрососудистым осложнением. В связи с тяжестью, недостаточной эффективностью и высокой стоимостью лечения терминальных стадий ХБП актуальность изучения эпидемиологических характеристик данной патологии приобретает ключевое значение.

Определение ХБП, первоначально сформулированHoe KDOQI (NKF, 2002) (National Kidney Foundation) [1] и впоследствии дополненное KDIGO (KDIGO, 2012) (Kidney Disease Improving Global Outcomes) [2], обобщает повреждение почек или снижение скорости клубочковой фильтрации (СКФ) менее 60 мл/мин/1,73 м², персистирующее в течение более 3 мес, независимо от первичного диагноза. Это определение, принятое нефрологическим сообществом, хорошо служит общественному здравоохранению. Определение ХБП требует 2-кратного измерения СКФ или альбуминурии (АУ) или двух показателей в течение как минимум 3 мес. Для надзора системы здравоохранения за ХБП одиночные измерения креатинина и АУ у пациентов в стабильных амбулаторных условиях представляются приемлемым компромиссом, обеспечивающим реалистическую оценку, несмотря на изменчивость этих показателей под влиянием ряда факторов (питание, физическая активность, состояние гидратации и др.). Следует обратить особое внимание на то, что популярные формулы расчета СКФ (Кокрофта-Голта, MDRD, (KD-EPI) имеют ограничения для очень пожилых людей, лиц с низкой мышечной массой, при крайних размерах

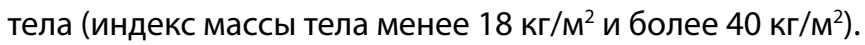

Для стабилизации числа пациентов с выраженными стадиями ХБП крайне важно раннее выявление боль-

Таблица 1. Стадии хронической болезни почек по уровню скорости клубочковой фильтрации [2]

\begin{tabular}{ccc}
\hline Стадия & Определение & $\begin{array}{c}\text { СКФ, мл/ } \\
\text { мин/1,73 м² }\end{array}$ \\
\hline 1 & Высокая и оптимальная & $>90$ \\
2 & Незначительно сниженная & $60-89$ \\
$3 а$ & Умеренно сниженная & $45-59$ \\
36 & Существенно сниженная & $30-44$ \\
4 & Резко сниженная & $15-29$ \\
5 & Терминальная почечная & $<15$ \\
\hline
\end{tabular}

ных с начальными стадиями и повсеместное внедрение в практику их лечения методов современной нефропротекции. Это снимает большие обременения заместительной почечной терапии (ЗПТ), необходимой на терминальной стадии ХБП.

\section{ЦЕЛЬ}

Оценить эпидемиологические характеристики развития ХБП у взрослых пациентов с СД 1 типа (СД1) и СД 2 типа (СД2) в динамике за период 2013-2016 гг.

\section{МЕТОДЫ}

\section{Дизайн исследования}

Объектом исследования является база данных Федерального регистра СД (ФРСД) 81 региона РФ, включенных в систему онлайн-регистра. Оценивались показатели распространенности и заболеваемости (новые случаи/год) на 10 тыс. взрослых больных СД (>18 лет) за 2013-2016 гг.

Анализ структуры терапии при ХБП проводился за 2017 г. (так как данные поля были введены в 2015 г.) по данным 10 лучших регионов по качеству ведения регистра. Данный подход использован с целью снижения влияния на полученные результаты фактора ведения регистра, поскольку поле антигипертензивной и другой сопутствующей терапии не позиционировалось в качестве обязательного и заполнялось качественно только в отдельных регионах.

Данные регистра позволили оценить не только уровень АУ, но и провести автоматический расчет СКФ по фор-

Таблица 2. Классификация хронической болезни почек по уровню альбуминурии [2]

\begin{tabular}{|c|c|c|c|c|}
\hline \multirow{2}{*}{$\begin{array}{l}\text { Кате- } \\
\text { гория }\end{array}$} & \multicolumn{2}{|c|}{ А/Кр мочи } & \multirow{2}{*}{$\begin{array}{c}\text { CЭА, } \\
\text { мг/24 час }\end{array}$} & \multirow{2}{*}{ Описание } \\
\hline & мг/ммоль & $\mathrm{M \Gamma} / \Gamma$ & & \\
\hline $\mathrm{A} 1$ & $<3$ & $<30$ & $<30$ & $\begin{array}{c}\text { Норма или } \\
\text { незначительно } \\
\text { повышена }\end{array}$ \\
\hline $\mathrm{A} 2$ & $3-30$ & $30-300$ & $30-300$ & $\begin{array}{l}\text { Умеренно } \\
\text { повышена }\end{array}$ \\
\hline A3 & $>30$ & $>300$ & $>300$ & $\begin{array}{c}\text { Значительно } \\
\text { повышена * }\end{array}$ \\
\hline
\end{tabular}

Примечания: *Включая нефротический синдром (СЭА >2200 мг/24 ч [А/Кр >2200 мг/г; >220 мг/ммоль]) 


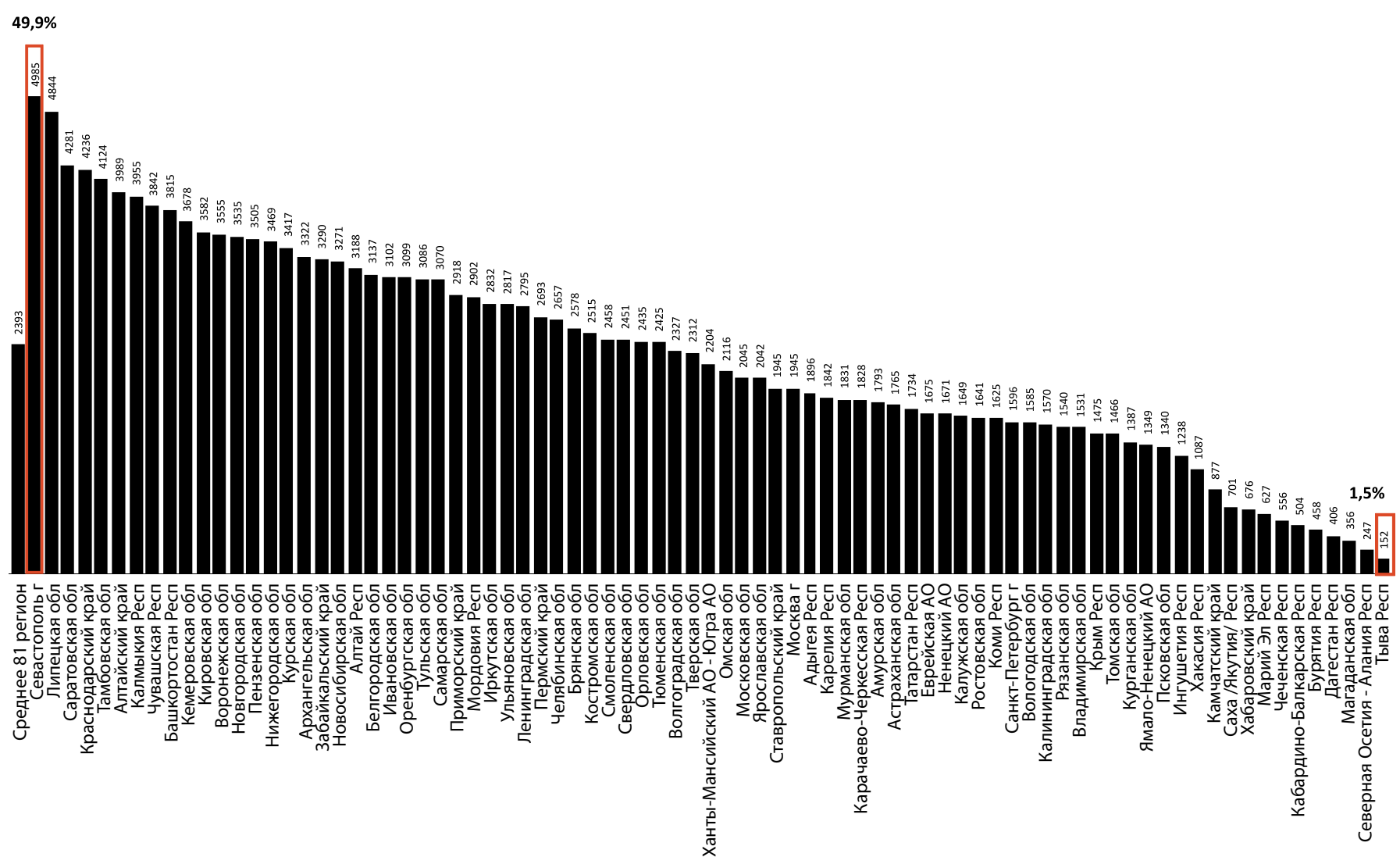

Рис. 1. Распространенность хронической болезни почек в регионах Росийской Федерации (на 10 тыс. взрослых пациентов с сахарным диабетом 1 типа), данные Федерального регистра сахарного диабета, 81 регион Российской Федерации, 2016 г.
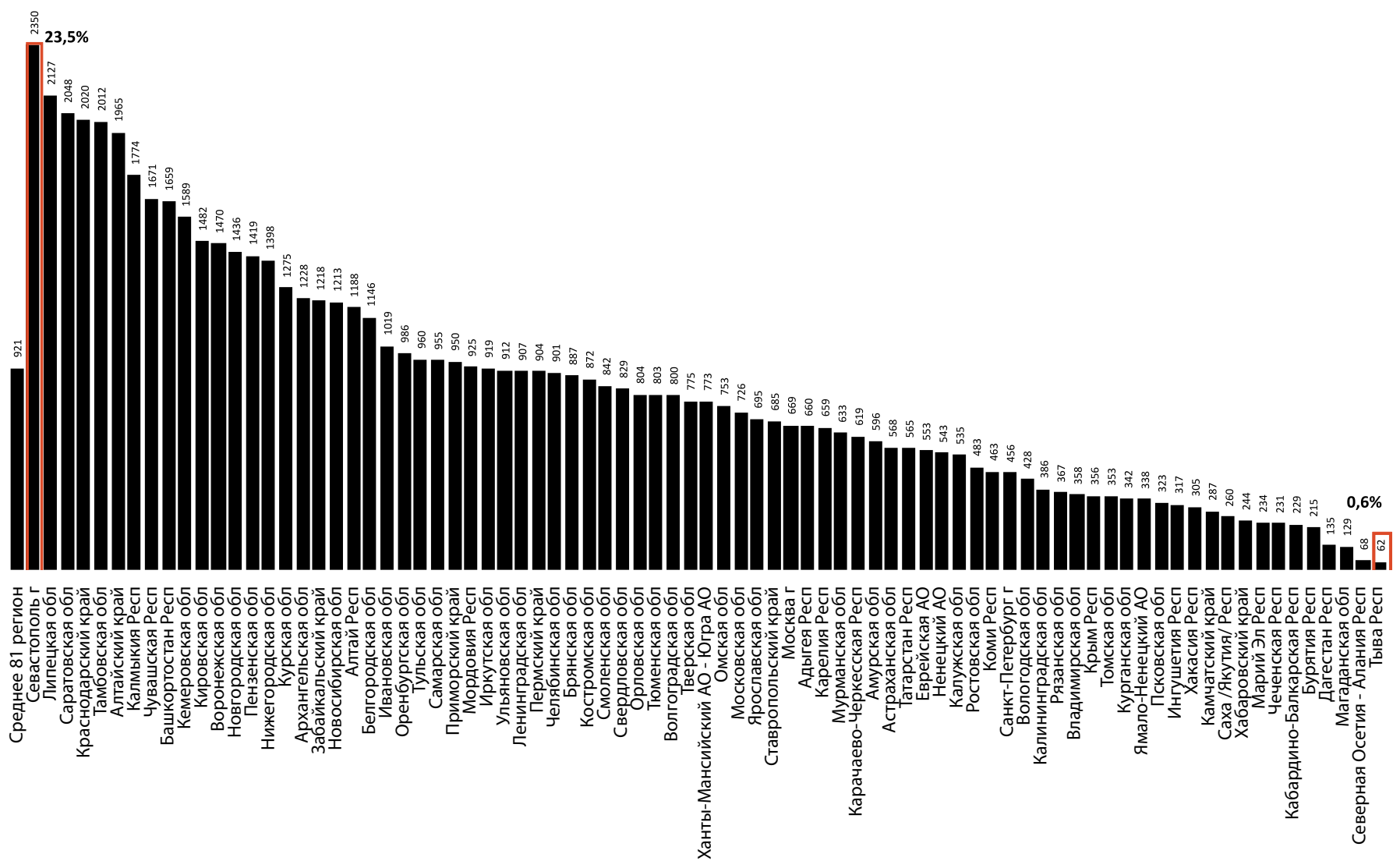

Рис. 2. Распространенность хронической болезни почек в регионах Росийской Федерации (на 10 тыс. взрослых пациентов с сахарным диабетом 2 типа), данные Федерального регистра сахарного диабета, 81 регион Российской Федерации, 2016 г.

муле CKD-EPI, что позволяет установить диагноз ХБП в соответствии с современными стандартами диагностики.

Согласно концепции ХБП, оценка стадии почечной патологии осуществляется по величине СКФ, признанной в качестве параметра, наиболее полно отражающего" количество и суммарный объем работы нефронов, в том числе связанной с выполнением неэкскреторных функций (табл. 1).

Методом количественной оценки АУ, не подверженным влиянию гидратации, служит измерение отношения альбумин/креатинин (A/Кр) в нефиксированном по времени про- 


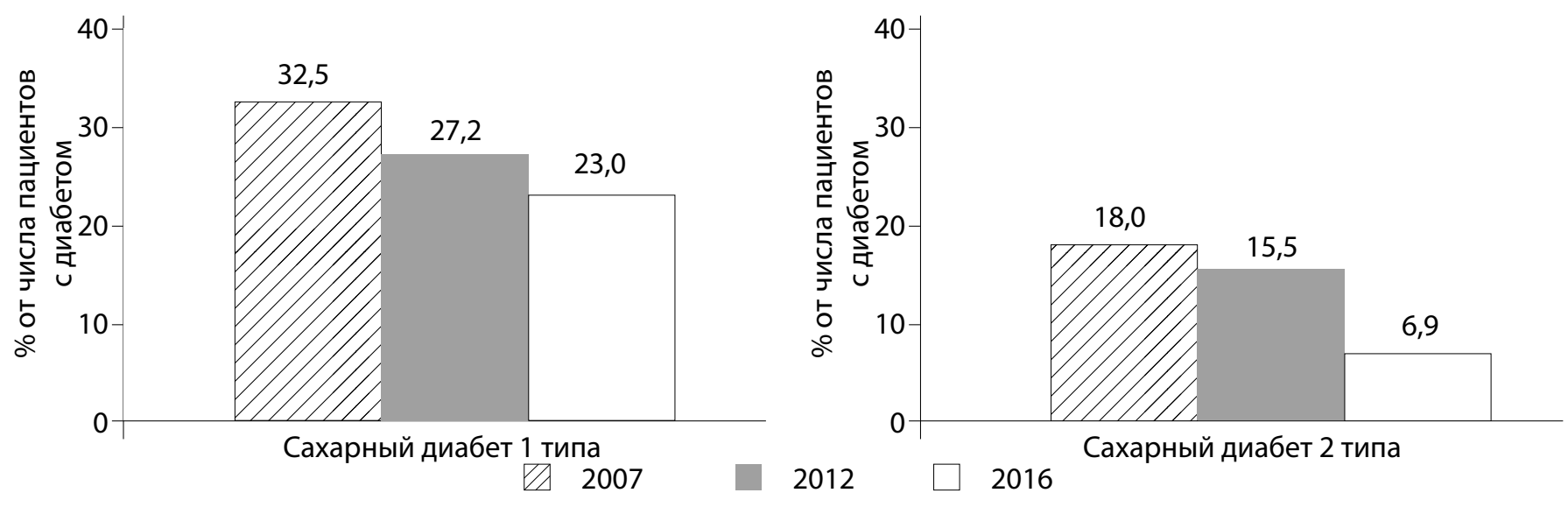

Рис. 3. Динамика распространенности диабетической нефропатии, хронической болезни почек (\% пациентов) при сахарном диабете 1 и 2 типа по данным Федеральной целевой программы в 2007 г., 2012 г. и Федерального регистра сахарного диабета в 2016 г.

Сахарный диабет 1 типа

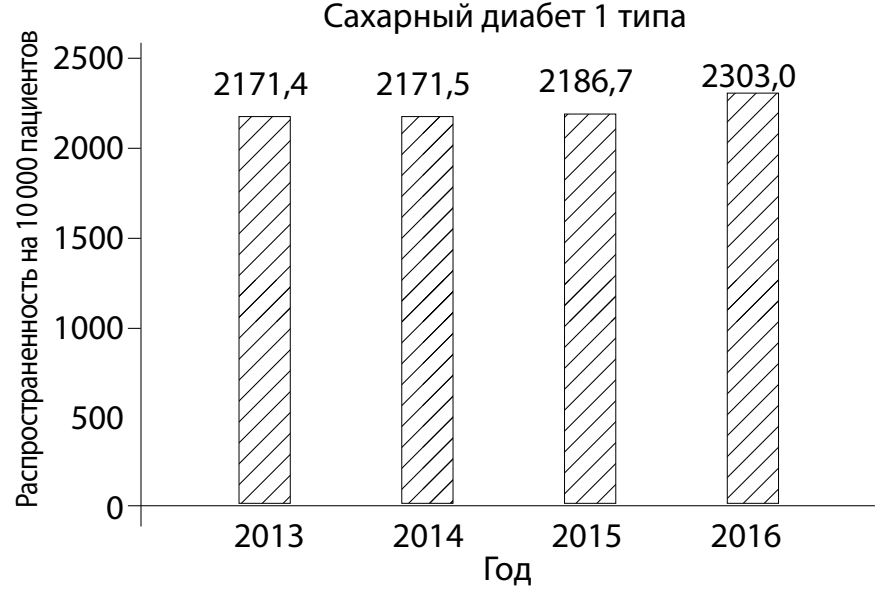

Сахарный диабет 2 типа

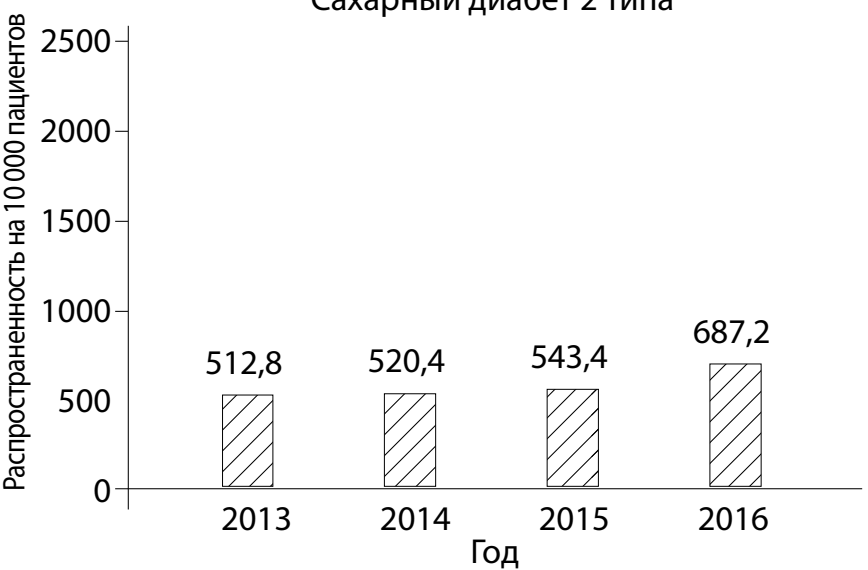

Рис. 4. Распространенность хронической болезни почек (на 10 тыс. взрослых пациентов) при сахарном диабете 1 и 2 типа в $2013-2016$ гг. по данным Федерального регистра сахарного диабета (81 регион Российской Федерации).
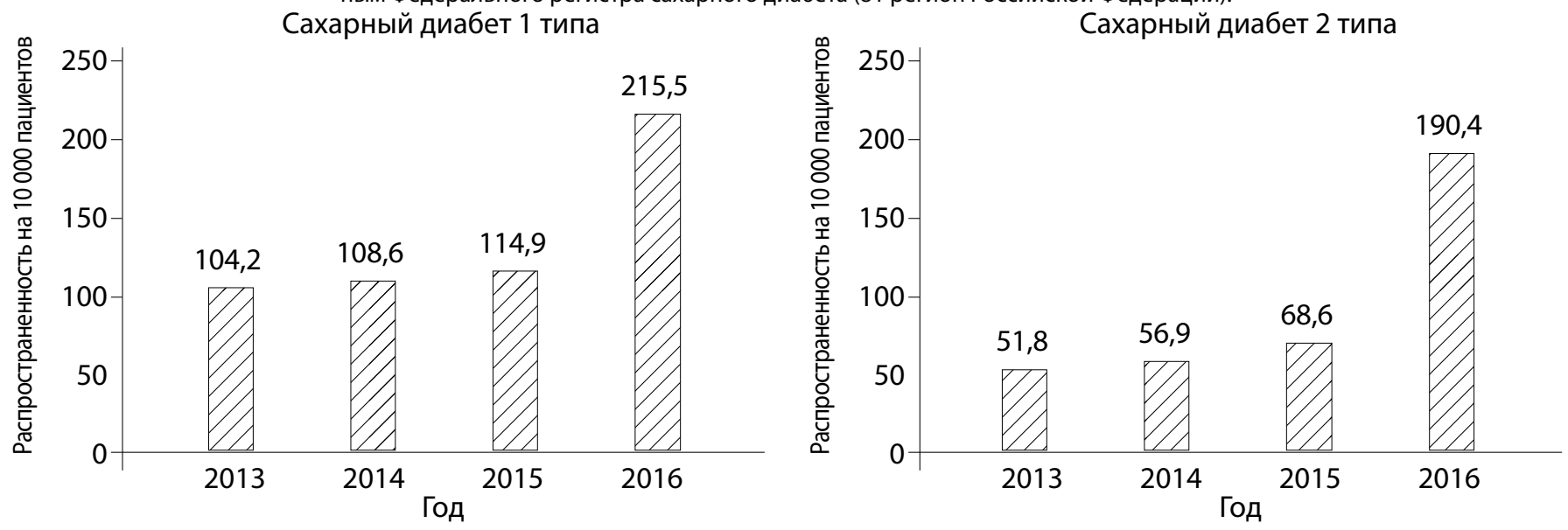

Рис. 5. Частота новых случаев хронической болезни почек в год на 10000 взрослых пациентов с сахарным диабетом 1 и 2 типа в динамике 2013-2016 гг., данные Федерального регистра сахарного диабета, 81 регион Российской Федерации.

извольном образце мочи. Предпочтительна первая утренняя порция мочи, наилучшим образом коррелирующая с 24-часовой экскрецией белка. Выделяют три категории АУ (табл. 2).

\section{Этическая экспертиза}

Протокол исследования № 20 от 14 декабря 2016 г. был рассмотрен этическим комитетом ФГБУ «НМИЦ эндокринологии» МЗ РФ, принято положительное решение.

\section{РЕЗУЛЬТАТЬ}

В 2016 г. частота регистрации ХБП (все стадии) в базе ФРСД в среднем по России составила 23\% при СД1 и 6,9\% при СД2, при этом отмечались выраженные межрегиональные различия в частоте регистрации ХБП от 1,5\% до 49,9\% при СД1 и от 0,6\% до 23,5\% при СД2 (рис. 1 и 2).

Динамика распространенности диабетической нефропатии, ХБП по данным ФРСД за 2016 г. по сравнению с данными, полученными в период осуществления подпрограммы «Сахарный диабет» Федеральной целевой программы «Предупреждение и борьба с социально-значимыми заболеваниями 2007-2012 гг.» (ФЦП), представлена на рис. 3.

Динамика осложнения, представленная на рис. 4, показывает рост распространенности ХБП в РФ в 1,1 раза при СД1 и в 1,3 раза при СД2 за последние 4 года по данным ФРСД. 

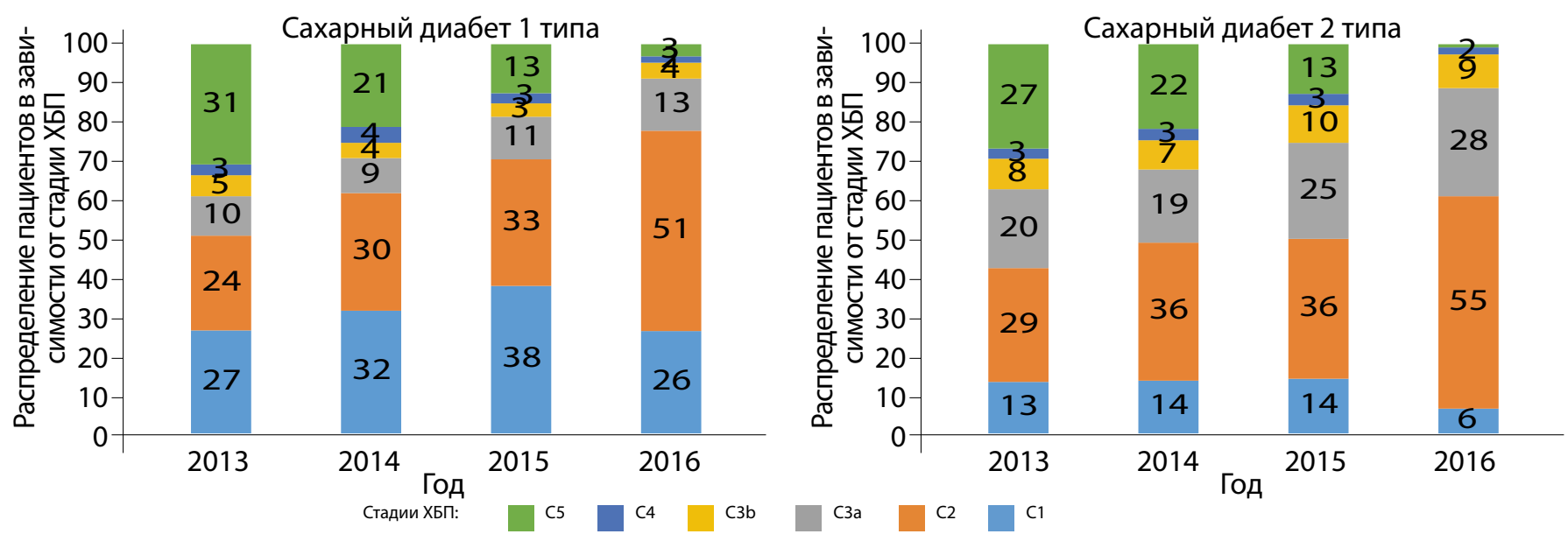

Рис. 6. Распределение по стадиям хронической болезни почек (новые случаи в год, \% пациентов) у взрослых с сахарным диабетом 1 и 2 типа в 2013-2016 гг. по данным Федерального регистра сахарного диабета, 81 регион Российской Федерации.
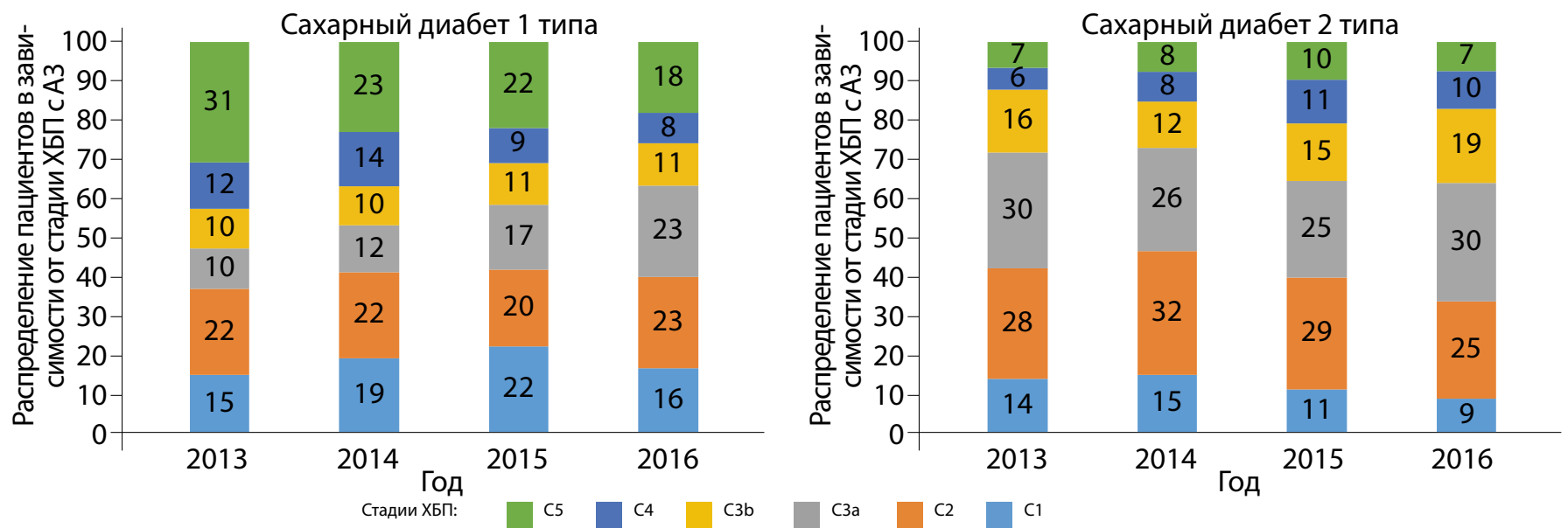

Рис. 7. Распределение по стадиям хронической болезни почек у пациентов с уровнем альбуминурии АЗ (новые случаи в год, \% пациентов) у взрослых с сахарным диабетом 1 и 2 типа в 2013-2016 гг. по данным Федерального регистра сахарного диабета, 81 регион Российской Федерации.
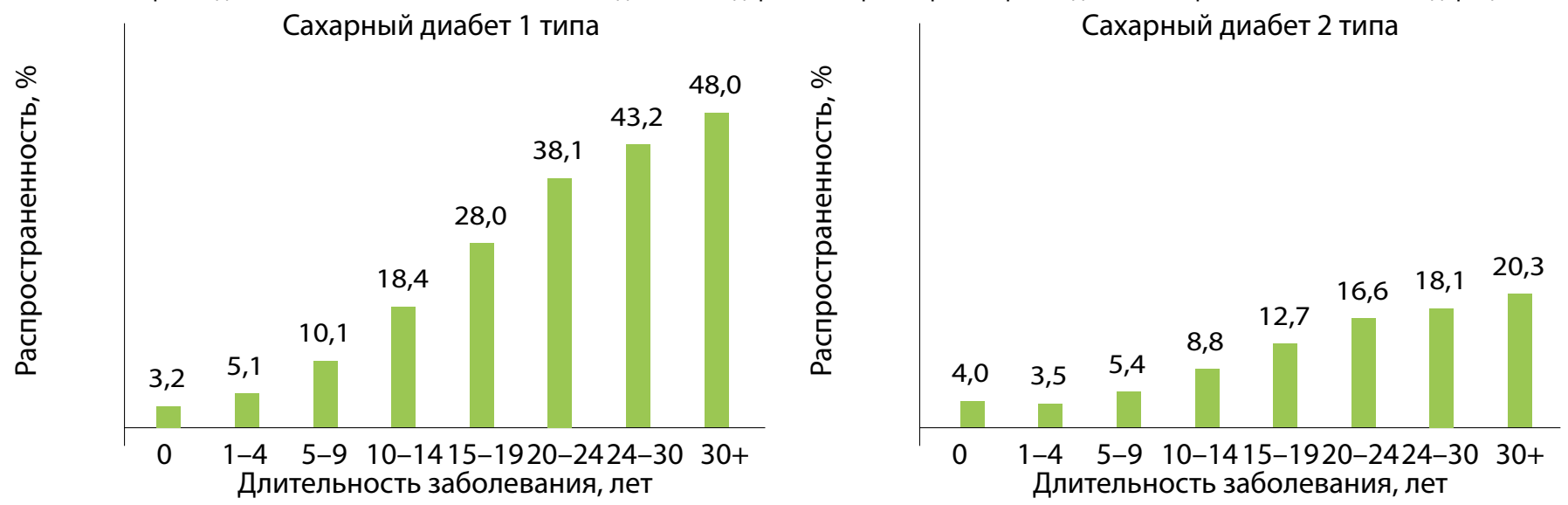

Рис. 8. Распространенность хронической болезни почек в зависимости от длительности сахарного диабета 1 и 2 типа, по данным Федерального регистра сахарного диабета, 81 регион Российской Федерации.

Частота регистрации новых случаев (заболеваемости) ХБП в ФРСД значимо возросла в 2016 г. по сравнению с 2013 г.: в 2 раза при СД1 и в 3,7 раза при СД2 (рис. 5).

При общем росте распространенности ХБП анализ распределения по стадиям указывает на улучшение диагностики осложнения - более раннее выявление на начальных стадиях ХБП С1-2 (почти в 2 раза) и значительно реже - на терминальной стадии (рис. 6).

Распределение ХБП по стадиям у пациентов с наличием АУ уровня АЗ (более 300 мг/сут или 200 мг/л или соотношение альбумин/креатинин в порции мочи более $300 \mathrm{mr/r)} \mathrm{представлено} \mathrm{на} \mathrm{рисунке} \mathrm{7,} \mathrm{также} \mathrm{указывает}$ на сокращение доли терминальной стадии С5 за последние 4 года.
Установлена зависимость распространенности ХБП от длительности диагноза СД. При длительности СД1 $<5$ лет ХБП развивается у 5,1\% пациентов, при длительности СД1 >30 лет - у 48,0\%, для пациентов С СД2: 3,5\% и 20,3\% соответственно (рис. 8).

Средний возраст дебюта ХБП у лиц с СД1 увеличился на 4,3 года до 40,4 года (в 2013 г. - 36,1), с СД2 - на 2,4 года до 66,9 года (64,5 $\rightarrow 66,9$ лет). Средняя длительность СД1 до развития ХБП возросла с 11,8 до 14,2 года, длительность СД2 до момента диагностики ХБП увеличилась с 7,6 до 8,2 года (рис. 9).

В структуре ХБП в динамике с 2013 г. до 2016 г. выявлено увеличение доли пациентов с низким и умеренным комбинированным риском сердечно-сосудистых 


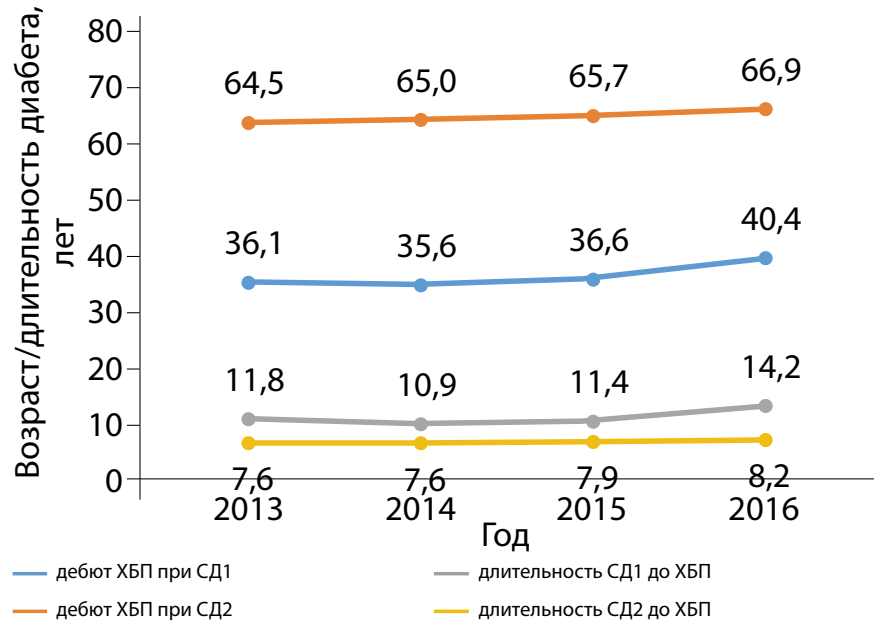

Рис. 9. Средний возраст и длительность сахарного диабета у пациентов до развития хронической болезни почек в 2013-2016 гг. по данным

Федерального регистра сахарного диабета, 81 регион Российской Федерации.

событий и терминальной почечной недостаточности (ТПН) по критериям KDIGO [2] с начальными стадиями ХБП С1-2, А1-2 - с 12,0\% до 46,8\% при СД1, с 10,0\% до 50,4\% при СД2. И напротив, доля пациентов с очень Высоким риском (стадии ХБП С3-5, А2-3) прогрессивно снижается: с $13,4 \%$ до 6,7\% при СД1, с 11,3 до 4,4\% при СД 2 (рис. 10).

Регистр располагает сведениями о применении препаратов нефропротективной направленности (для лечения артериальной гипертензии (АГ), дислипидемии, коррекции анемии) (табл. 3). В настоящее время данные поля в регистре не являются обязательными для заполнения, поэтому в анализ включены данные 10 регионов РФ, ведущих по качеству ввода данных в ФРСД.

АГ является наиболее значимым осложнением ХБП и лидирующим фактором ее прогрессирования. Распределение по уровню АД в зависимости от наличия ХБП у пациентов с СД1 и СД2 в 81 регионе по данным ФРСД в динамике 2013-2017 гг. представлено в табл. 4. Градация АД была проведена в соответствии с рекомендуемым целевым уровнем для пациентов с наличием ХБП (<130/85 мм рт.ст.), стандартным целевым уровнем у пациентов с СД (<140/85 мм рт.ст.) и неудовлетворительным контролем АГ ( $\geq 140$ /85 мм рт.ст.).

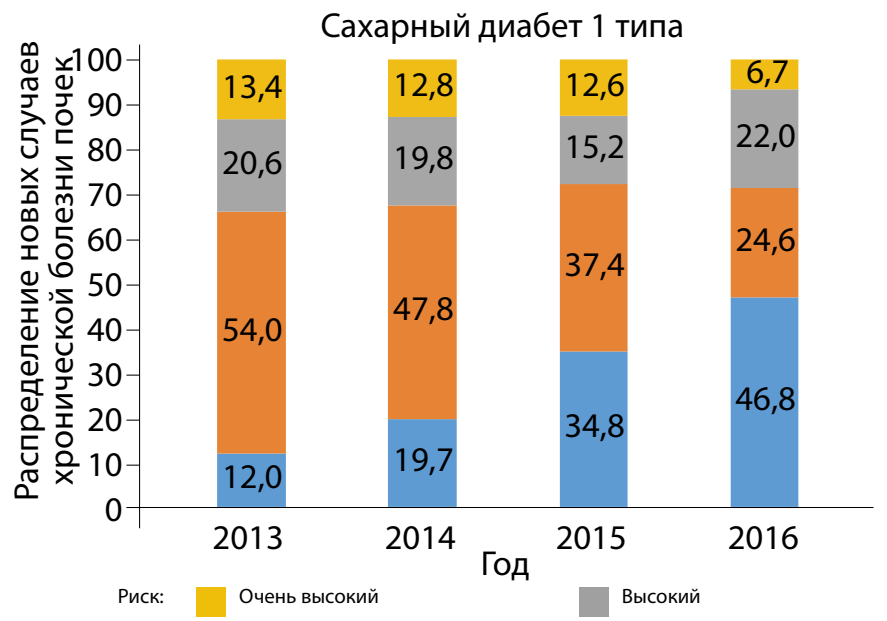

\section{ОБСУЖДЕНИЕ}

При анализе динамики распространенности ХБП в период 2013-2016 гг. следует учитывать несколько факторов, важнейшим из которых является изменение парадигмы диагностики осложнения. Именно в данный период произошел переход от оценки АУ в качестве классического маркера диабетической нефропатии к учету уровня СКФ для верификации ХБП как наднозологического состояния [3]. Огромный пул пациентов со снижением СКФ при нормоальбуминурии, который не учитывался в диагнозе диабетической нефропатии, увеличил численность лиц с этим осложнением по критериям ХБП. С этим может быть связан существенный рост показателя распространенности ХБП за последние 4 года при отсутствии истинного повышения частоты развития осложнения, на что указывает сравнительный анализ с данными ФЦП 2007-2012 гг. [4].

Кроме того, изменение ряда технических параметров регистра, а именно внедрение расчета СКФ по формуле CKD-EPI вместо формулы Кокрофта-Голта (завышающей результат), которое произошло в 2015 г., позволило более точно оценивать наличие и стадию ХБП. Для определенных категорий лиц оценка СКФ расчетными методами, имеющими ограничения при морбидном ожирении, возрасте более 85 лет, дефиците массы тела, трансплантированной почке, могло повлиять на данные статистики, поскольку в этих случаях расчетная СКФ, как правило, ниже ее истинных значений.

Тем не менее выраженные межрегиональные различия в частоте диагностики осложнения могут свидетельствовать о сохраняющихся проблемах скрининга ХБП в ряде регионов, где не выполняется стандарт обследования пациентов с СД с обязательной оценкой СКФ и АУ не реже 1 раза в год $[5,6]$. Наиболее ярко это проявляется в явно заниженной диагностике ХБП при СД2 (6,3\% в среднем по РФ), особенно по сравнению с результатами клинико-эпидемиологических исследований с активным скринингом, где этот показатель достигал 40-60\% [4, 7].

Особого внимания заслуживает факт нормоальбуминурии при сниженной СКФ у большого числа пациентов, особенно при СД2. Возможно, изменилось «лицо» диабетической нефропатии, и мы уходим от альбуминцентрических представлений, рассматривающих АУ в качестве

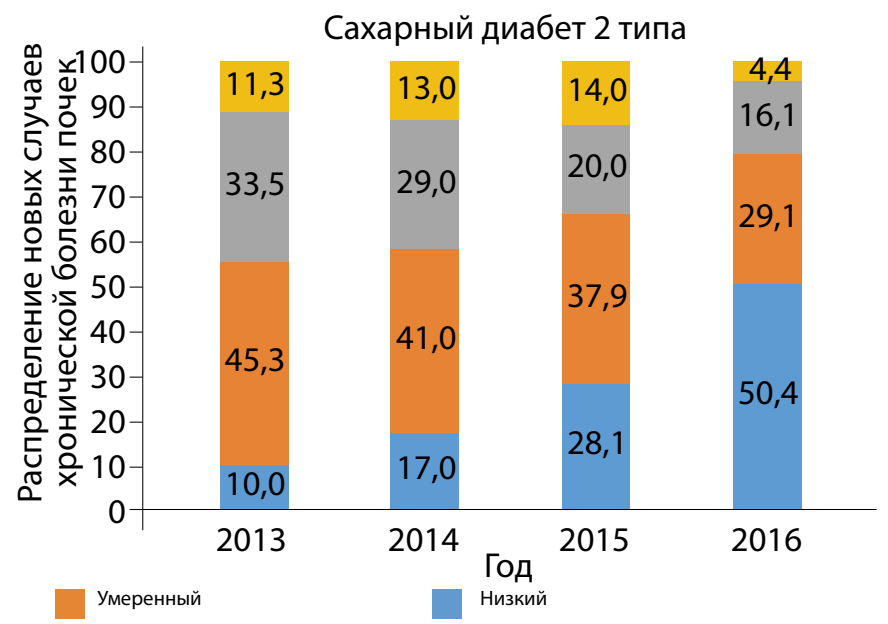

Рис. 10. Распределение пациентов с хронической болезнью почек (новые случаи/год, \% пациентов) по критериям KDIGO риска сердечно-сосудистых событий и терминальной почечной недостаточности у взрослых пациентов с сахарным диабетом 1 и 2 типа в $2013-2016$ гг. по данным Федерального регистра сахарного диабета, 81 регион Российской Федерации. 
Таблица 3. Структура антигипертензивной, гиполипидемической, антиагрегантной и антианемической терапии, 2017 г., 10 регионов Российской Федерации

\begin{tabular}{|c|c|c|c|c|c|c|c|c|}
\hline \multirow{3}{*}{$\begin{array}{c}\text { Тип СД } \\
\text { Наличие ХБП } \\
\text { Группы препаратов }\end{array}$} & \multicolumn{4}{|c|}{ Сд 1 типа } & \multicolumn{4}{|c|}{ СД 2 типа } \\
\hline & \multicolumn{2}{|c|}{ Нет ХБП } & \multicolumn{2}{|c|}{ ХБП* } & \multicolumn{2}{|c|}{ Нет ХБП } & \multicolumn{2}{|c|}{ ХБП ${ }^{*}$} \\
\hline & $\begin{array}{c}\text { \% от } \\
\text { группы } \\
\text { без } \\
\text { ХБП }\end{array}$ & $\begin{array}{c}\text { \% от } \\
\text { группы } \\
\text { на те- } \\
\text { рапии }\end{array}$ & $\begin{array}{c}\text { \% от } \\
\text { группы } \\
\text { с ХБП }\end{array}$ & $\begin{array}{c}\text { \% от } \\
\text { группы } \\
\text { на те- } \\
\text { рапии }\end{array}$ & $\begin{array}{c}\text { \% от } \\
\text { группы } \\
\text { без } \\
\text { ХБП }\end{array}$ & $\begin{array}{l}\text { \% от } \\
\text { группы } \\
\text { на те- } \\
\text { рапии }\end{array}$ & $\begin{array}{l}\text { \% от } \\
\text { группы } \\
\text { с ХБП }\end{array}$ & $\begin{array}{c}\text { \% от } \\
\text { группы } \\
\text { на те- } \\
\text { рапии }\end{array}$ \\
\hline Антигипертензивная терапия & 8,4 & & 24,7 & & 23,6 & & 45,6 & \\
\hline Ингибиторы АПФ & 5,9 & 69,5 & 18,0 & 72,9 & 15,8 & 66,9 & 32,9 & 72,2 \\
\hline Блокаторы рецепторов ангиотензина II (БРА) & 1,2 & 13,8 & 4,3 & 17,5 & 4,6 & 19,2 & 14,8 & 32,4 \\
\hline Диуретики & 2,9 & 34,1 & 8,6 & 34,9 & 11,2 & 47,3 & 26,2 & 57,5 \\
\hline Бета-блокаторы (ББ) & 2,4 & 28,4 & 8,7 & 35,4 & 8,6 & 36,5 & 22,4 & 49,1 \\
\hline Альфа-блокаторы & 0,0 & 0,4 & 0,2 & 0,7 & 0,1 & 0,4 & 0,2 & 0,4 \\
\hline Блокаторы кальциевых каналов & 0,7 & 8,1 & 3,8 & 15,5 & 3,8 & 15,9 & 14,3 & 31,5 \\
\hline Препараты центрального действия & 0,1 & 0,6 & 0,4 & 1,8 & 0,2 & 1,0 & 1,1 & 2,4 \\
\hline Прямые ингибиторы ренина & 0,0 & 0,4 & 0,0 & 0,1 & 0,0 & 0,2 & 0,0 & 0,1 \\
\hline Гиполипидемические препараты & 3,9 & & 12,8 & & 10,4 & & 28,4 & \\
\hline Статины & 3,6 & 94,4 & 10,9 & 85,1 & 10,1 & 97,2 & 27,1 & 95,5 \\
\hline Фибраты & 0,2 & 5,6 & 2,2 & 17,2 & 0,1 & 1,4 & 2,0 & 6,9 \\
\hline Антиагреганты & 2,8 & & 9,2 & & 10,1 & & 26,1 & \\
\hline Антианемические препараты & 0,1 & & 1,8 & & 0,2 & & 1,0 & \\
\hline
\end{tabular}

Примечание: *ХБ высоких градаций - А2 или АЗ и/или С3-5

Таблица 4. Распределение по уровню артериального давления (мм рт.ст.) в зависимости от наличия хронической болезни почек при сахарном диабете 1 и 2 типа (\% пациентов) в 81 регионе по данным Федерального регистра сахарного диабета

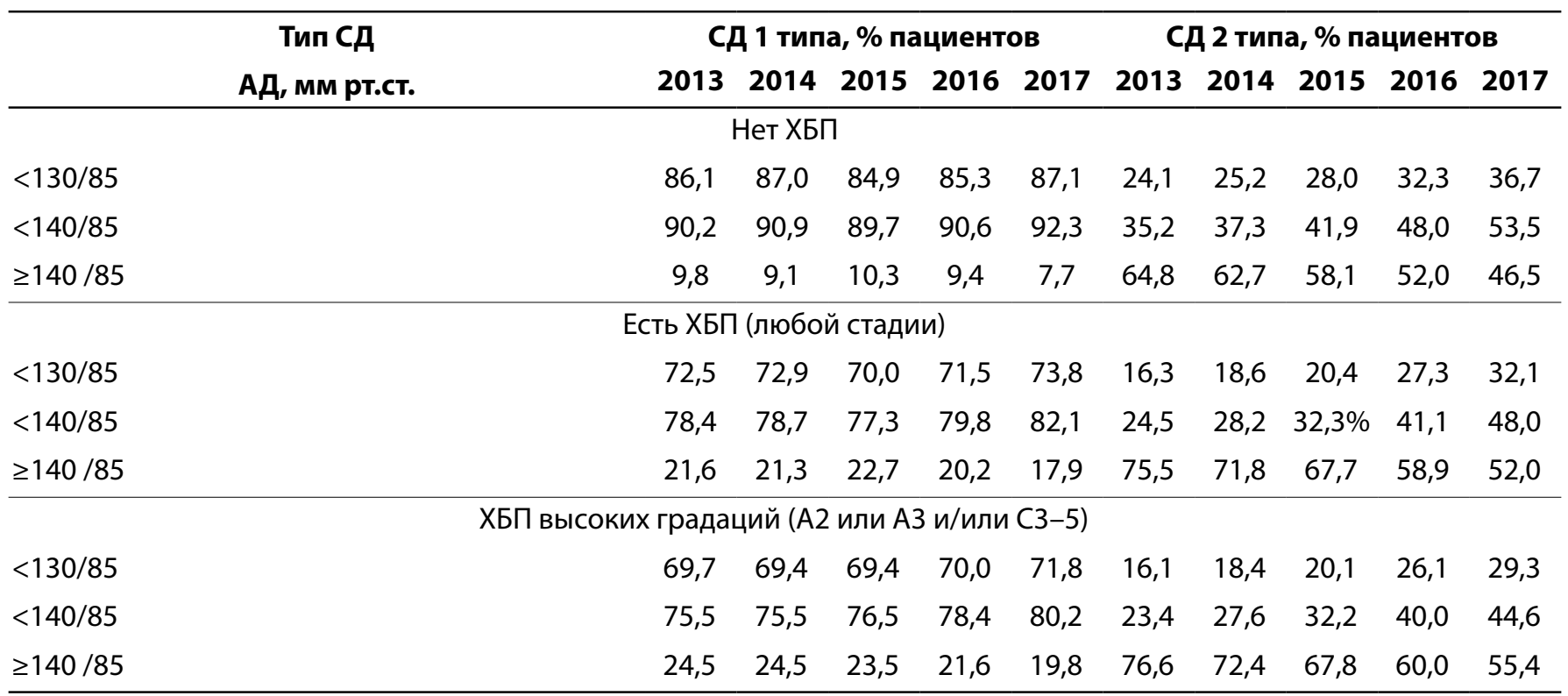

показателя манифестации патологического процесса, ведущего к протеинурии, последующему снижению СКФ и, в конечном итоге, к ТПН. Показательны данные США с 1988 по 2014 гг. среди 6251 взрослых пациентов с СД старше 20 лет - участников программы NHANES (National Health and Nutrition Examination Survey), у которых определялись АУ и снижение СКФ <60 и 30 мл/мин/1,73 м² [8]. Распространенность ХБП значимо не менялась (с 28\% до 26\%), при этом распространенность АУ прогрессивно снижалась с годами с $20 \%$ до $15,9 \%$ (р<0,001), в то вре- мя как снижение СКФ возрастало с 9\% до 14\%, особенно $<30$ мл/мин/1,73 м². Снижение частоты АУ оказалось характерным для лиц моложе 65 лет и белых, для СКФ зависимости от возраста и расы не обнаружено. В случае АУ эти результаты можно объяснить улучшением контроля гликемии, АД, липидов, широким использованием блокады ренин-ангиотензин-альдостероновой системы (PAAC). В случае снижения СКФ этих объяснений недостаточно. Возраст пациентов существенно не менялся, но увеличивалась длительность СД. В определенной сте- 
пени, увеличение продолжительности жизни пациентов в комбинации со снижением смертности от кардиоваскулярных заболеваний и других осложнений диабета дают возможность и время для развития прогрессивных повреждений в почках.

Учитывая современные тенденции, представляется важным оценивать среднюю СКФ по годам, особенно у пациентов в возрасте старше 60 лет, поскольку в этой возрастной группе отмечается наибольшая распространенность ХБП. Так, средняя СКФ для лиц старше 60 лет в США по данным USRDS (United States Renal Data System) составляет 25 мл/мин/1,73 м², что ниже, чем средняя СКФ для всей выборки с ХБП [9].

Данные ФРСД показали, что успехи в ведении пациентов с СД в последние годы не снижают риск ХБП, но дают отсрочку в ее развитии. Характерно увеличение возраста и длительности заболевания к моменту диагностики осложнения. К сожалению, мы не располагаем точными данными о лечении терминальных стадий, поэтому достаточно сложно оценить показатели Российского регистра заместительной почечной терапии, согласно которым, в структуре причин ТПН у пациентов, получающих лечение программным гемодиализом, перитонеальным диализом и с функционирующим почечным трансплантатом пациенты с СД составляют 13,8\%, 14,6\% и 5,8\% соответственно [10].

Эпидемиологическое исследование Pittsburgh Epidemiology of Diabetes Complications (Pittsburgh EDC), оценившее 50-летний риск развития почечных осложнений у 932 пациентов с дебютом СД1 в детском возрасте в 1950-1980 гг., показало, что этот риск не снижается (независимо от пола) при наиболее низком среди лиц, заболевших в раннем детском возрасте (до 6 лет) [11]. Результаты исследования показали влияние длительного течения СД1 на риски выраженных стадий ХБП. При длительности СД1 более 40 лет показаны обнадеживающие данные о снижении частоты терминальной стадии ХБП на 45\% при росте микроальбуминурии на 3\% и стабильной частоте макроальбуминурии. Эти результаты, как считают эксперты, отражают улучшение подходов к лечению ХБП и, как следствие, ведут к более медленному прогрессированию осложнения, но не предупреждают его развитие. Таким образом, увеличение продолжительности жизни пациентов с СД1 и патологией почек ставит в полный рост вопросы профилактики, ориентированной не только на гликемический контроль, но и на управление другими факторами риска (дислипидемией, гипертонией), а также поиск дополнительных целей терапевтического воздействия.

Данные национального норвежского регистра детского диабета свидетельствуют о возрастающей частоте развития ТПН среди 7871 пациентов с дебютом СД1 в детском возрасте: 0,7\% при длительности заболевания 20 лет, 2,9\% - 30 лет, 5,3\% - 40 лет. Риск развития был ниже у женщин, чем у мужчин (0,62; 95\% ДИ 0,41-0,91) [12].

Близкие данные получены в когортном исследовании в Финляндии, включавшем 29906 пациентов с диагнозом СД1 в возрасте до 30 лет в 1965-2011 гг. с наблюдением до начала 3ПТ, смерти или завершения исследования в 2013 г. ТПН развилась у 2,2\% пациентов после 20 лет и у 7\% - после 30 лет длительности заболевания [13].

Несомненно, особого внимания требуют лица с дебютом СД в период пубертата. По данным упомянутого норвежского регистра, имеется более высокий риск развития ТПН у лиц с дебютом СД в возрасте 10-14 лет, по сравнению с возрастом дебюта до 10 лет (1,29; 95\% ДИ 1,06-1,56) [12].

Пубертат - период, когда чрезвычайно сложна задача достижения компенсации. В это время многочисленные факторы конкурируют за внимание к ним, а вопросы контроля СД занимают весьма скромное место в списке приоритетов [14], что может способствовать раннему развитию осложнений до стандартного времени первичной диагностики.

Обращает на себя внимание факт развития ХБП по данным ФРСД у 5,1\% взрослых пациентов при длительности СД1 менее 5 лет. Возможно, стоит изменить общепринятый стандарт начала обследования через 5 лет от дебюта СД1, чтобы не упустить эти 5,1\% пациентов? С другой стороны, под маской диабетической болезни почек может скрываться другая почечная патология, прежде всего, мочевая инфекция.

Регистрация заболеваемости ХБП у взрослых пациентов, по данным ФРСД, значимо возросла в 2016 г. по сравнению с 2013 г.: в 2 раза при СД1 и в 3,7 раза при СД2. При общем росте распространенности ХБП анализ распределения по стадиям указывает на улучшение диагностики осложнения. ХБП - «немое» заболевание на начальных стадиях, и немногие пациенты догадываются о его развитии на стадиях 1-3. Для уменьшения количества пациентов, прогрессирующих до выраженных стадий ХБП, крайне важно раннее выявление больных с начальными стадиями и повсеместное внедрение в практику их лечения методов современной нефропротекции. Следует отметить более раннюю диагностику осложнения на стадии 2 (почти в 2 раза)и значительное снижение на терминальной стадии ХБП. Значительно реже это происходит на терминальной стадии ХБП. Такая положительная динамика должна повлиять на численность пациентов с СД, начинающих диализ с поздней диагностикой ТПН, пройдя жизнеспасающую терапию в реанимации [10].

Заболеваемость с учетом АУ уровня АЗ, свидетельствующей о развитии выраженной ХБП и ее поздней диагностике у лиц с более низкой функцией почек, в динамике с 2013 г. по 2016 г. пока не снижается, что должно стать предметом особого внимания и анализа. С другой стороны, это может отражать долю истинной диабетической нефропатии с преимущественным поражением почечного фильтра в структуре ХБП, диагностированной по новым стандартам.

В регистр поступают сведения о применении препаратов нефропротективной направленности (для лечения АГ, дислипидемии, коррекции анемии). Пока нет сведений о медикаментах для лечения минеральных и костных нарушений при ХБП. Регистр содержит только обобщенные данные, однако даже в таком варианте сведения о лекарственной терапии пациентов позволяют оценить текущую практику лечения важных клинических синдромов. Данные показывают более частое назначение при наличии ХБП антигипертензивной терапии и преобладание в ее структуре препаратов нефропротективного ряда (ингибиторы ангиотензинпревращающего фермента (ИАПФ) и блокаторы рецепторов ангиотензина II (БРА)), что указывает на клиническую значимость данного состо- 
яния для назначения терапии и ее выбора. ИАПФ и БРА являются опорой нефропротективной терапии. При этом их использование, особенно при ХБП высоких градаций (А2 или А3 и/или С3-5), к сожалению, далеко от стандарта - не превышает 50\% при СД2 и 22,3\% при СД1 (табл. 3). Такая же тенденция отмечается и в отношении другой органопротективной терапии (гиполипидемической, антианемической). Учет назначений данной терапии стал возможен только с 2015 г. после включения в онлайн-регистр соответствующих полей. Остается надеяться, что сведения о нефропротективной терапии пока не внесены в регистр. Поэтому представленные данные о частоте назначения препаратов могут не в полной мере отражать реальную клиническую практику.

АГ, являясь наиболее значимым осложнением ХБП, играет ключевую роль в ее прогрессировании, как и в развитии макроваскулярной патологии, что требует оптимизации контроля АД. По мере прогрессирования ХБП роль метаболических факторов снижается и возрастает роль гемодинамических. Анализ данных распределения по уровню АД (целевой для пациентов с наличием ХБП, стандартный для пациентов с СД и неудовлетворительный) показал позитивные тенденции: рост числа пациентов с ХБП, включая высокие градации, достигших уровня АД менее 130/85 мм рт. ст., признанного безопасным [5] для дальнейшего прогрессирования поражения почек. К сожалению, основная часть пациентов остается вне целевого диапазона, особенно при СД2. Остаются актуальными рекомендации KDIGO, предлагающие индивидуализировать целевой уровень АД для пациентов с ХБП в зависимости от возраста, имеющейся сердечно-сосудистой патологии, сопутствующих заболеваний, риска прогрессирования ХБП, наличия или отсутствия ретинопатии и толерантности к проводимой терапии [2]. Тем не менее классически безопасным порогом АД для пациентов с ХБП является показатель $<130 / 85$ мм рт. ст. [5].

По ранее опубликованным данным ФРСД среди причин смерти пациентов с СД в РФ ведущие позиции продолжает занимать сердечно-сосудистая патология при обоих типах СД: суммарно хроническая сердечно-сосудистая недостаточность, нарушения мозгового кровообращения, инфаркт миокарда и острые сердечно-сосудистые нарушения являются причиной смерти 31,9\% пациентов с СД1 и 49,5\% пациентов с СД2, что подтверждает приоритет ранней диагностики и терапевтических подходов, направленных на коррекцию сердечно-сосудистых рисков у пациентов с СД [15].

ХБП была определена как независимый фактор риска сердечно-сосудистых заболеваний. Среди пациентов с ХБП смерть от кардиоваскулярной патологии происходит гораздо чаще, чем прогрессирование до терминальной стадии почечной недостаточности [16]. Сложные взаимоотношения между кардиоваскулярной патологией и заболеванием почек базируются на общности популяционных факторов риска. Наряду с традиционными факторами риска в условиях снижения почечной функции нетрадиционные (анемия, гиперпаратиреоз, дефицит витамина D, гипоальбуминемия, гиперфосфатемия, снижение СКФ, АУ и др.) ускоряют развитие сердечно-сосудистых заболеваний. Поэтому патофизиология последних представляется многогранной и уникальной в условиях прогрессирующей почечной дисфункции.
В структуре ХБП в динамике с 2013 г. до 2016 г. выявлено увеличение доли пациентов с низким и умеренным комбинированным риском сердечно-сосудистых событий и ТПН по критериям KDIGO, т.е. с начальными стадиями ХБП (С1-2, А1-2) и, напротив, прогрессивное снижение доли пациентов с очень высоким риском (стадии ХБП (3-5, А2-3). Несмотря на такое достижение, врачи должны оставаться бдительными к симптомам кардиоваскулярной дисфункции и быть готовыми к применению современных методов диагностики и лечения.

Что еще может представить ФРСД в перспективе?

1. Эпидемиологию сердечно-сосудистых заболеваний и кардиоваскулярную смертность в популяции пациентов с ХБП.

2. Оценку всех случаев смертности и госпитализации в зависимости от наличия и отсутствия ХБП.

3. Среднюю СКФ для лиц с ХБП старше 60 лет.

4. Полную фармакотерапию пациентов с ХБП, включая средства, стимулирующие эритропоэз, препараты железа, витамин D и его аналоги, фосфатбиндеры.

5. Число пациентов с ХБП, достигших целевого уровня контроля гликемии по $\mathrm{HbA}_{1 c^{\prime}}$ АД, липидного спектра, $\mathrm{Hb}$, показателей минерально-костных нарушений.

6. Динамику развития ХБП вплоть до ТПН и его осложнений (АГ, анемии, электролитных и минерально-костных нарушений и др.), инициацию ЗПТ, частоту ургентной госпитализации.

7. Сведения о курации эндокринологами пациентов на диализе, после трансплантации почки и сочетанной трансплантации почки и поджелудочной железы.

\section{ЗАКЛЮЧЕНИЕ}

При общем увеличении распространенности ХБП в Российской Федерации в динамике 2013-2016 гг. отмечается улучшение качества диагностики осложнения на более ранних стадиях с низким риском сердечно-сосудистых событий и ТПН, в более позднем возрасте и при большей длительности СД. Успехи в ведении пациентов с СД в последние годы не снижают риск ХБП, но дают отсрочку в ее развитии. Выраженные межрегиональные различия в частоте регистрации ХБП в ФРСД указывают на проблемы диагностики в ряде регионов, где не выполняется стандарт обследования пациентов с СД с обязательной оценкой СКФ и альбуминурии не реже 1 раза в год. Постоянный мониторинг региональных баз данных, повышение качества заполнения регистра позволят повысить качество получаемой информации и тем самым улучшить не только диагностику, но и перспективы лечения пациентов с данной патологией.

\section{ДОПОЛНИТЕЛЬНАЯ ИНФОРМАЦИЯ}

Источник финансирования. Работа проведена в рамках выполнения Государственного задания Министерства здравоохранения Российской Федерации о АAАA-A18-118051590061-9.

Конфликт интересов. Исаков М.А. является сотрудником ЗАО «Астон Консалтинг», обеспечивающего техническое сопровождение Федерального регистра сахарного диабета в on-line формате (кампания ЗАО «Астон Консалтинг» не являлась спонсором исследования, не принимала участие в анализе данных, их интерпретации и подготовке статьи, не состояла в финансовых отношениях с другими членами авторского коллектива). Осталь- 
ные авторы декларируют отсутствие явных и потенциальных конфликтов интересов, связанных с публикацией настоящей статьи.

Участие авторов: все авторы внесли значимый вклад в проведение исследования и подготовку статьи, прочли и одобрили финальную версию текста перед публикацией.
Благодарности. ЗАО «Астон Консалтинг» за техническое сопровождение регистра СД в онлайн-формате.

Всем медицинским специалистам (врачам, медицинским сестрам, регистраторам данных), ведущим активную работу по заполнению базы данных регистра СД.

\section{СПИСОК ЛИТЕРАТУРЫ | REFERENCES}

1. National Kidney F. KVOQI clinical practice guidelines for chronic kidney disease: evaluation, classification, and stratification. Am J Kidney Dis. 2002;39(2 Suppl 1):S1-266.

2. Kellum JA, Lameire N, Aspelin P, et al. Kidney disease: Improving global outcomes (KDIGO) acute kidney injury work group. KDIGO clinical practice guideline for acute kidney injury. Kidney Int Suppl. 2012;2(1):1-138. doi: 10.1038/kisup.2012.1

3. Дедов И.И., Шестакова М.В., Александров А.А., и др. Алгоритмы специализированной медицинской помощи больным сахарным диабетом (5-й выпуск) // Сахарный диабет. - 2011. - T. 14. - №3S. C. 2-72. [Dedov II, Shestakova MV, Aleksandrov AA, Galstyan GR, Grigoryan OR, Esayan RM, et al. Algorithms of Specialized Medical Care for Diabetes Mellitus Patients. Diabetes mellitus. 2011;14(3s):2-72. (In Russ.)] doi: 10.14341/2072-0351-5612

4. Дедов И.И., Шестакова М.В., Сунцов Ю.И., и др. Результаты реализации подпрограммы «Сахарный диабет» Федеральной целевой программы «Предупреждение и борьба с социально значимыми заболеваниями 2007-2012 годы» // Сахарный диабет. - 2013. - Т. 16. - №2S. C. 1-48. [Dedov II, Shestakova MV, Suntsov Yl, et al. Federal targeted programme "Prevention and Management of Socially Significant Diseases (2007-2012)": results of the "Diabetes mellitus" sub-programme. Diabetes mellitus. 2013;16(2S):1-48. (In Russ.)] doi: 10.14341/2072-0351-3879

5. Дедов И.И., Шестакова М.В., Майоров А.Ю., и др. Алгоритмы специализированной медицинской помощи больным сахарным диабетом / Под ред. Дедова И.И., Шестаковой М.В., Майорова А.Ю. - 8-й выпуск // Сахарный диабет. - 2017. - T. 20. - №1S. - C. 1-121. [Dedov II, Shestakova MV, Mayorov AY, et al. Dedov II, Shestakova MV, Mayorov AY, editors. Standards of specialized diabetes care. 8th edition. Diabetes mellitus. 2017;20(1S):1-121. (In Russ.)] doi: 10.14341/DM8146

6. American Diabetes Association. Standards of medical care in diabetes--2014. Diabetes Care. 2014;37 Suppl 1:S14-80. doi: 10.2337/dc14-S014

7. Маслова О.В., Сунцов Ю.И., Шестакова М.В., и др. Распространенность диабетической нефропатии и хронической болезни почек при сахарном диабете в Российской Федерации // Клиническая несрология. - 2010. - №3. - C. 45-50. [Maslova OV, Suntsov YI, Shesta- kova MV, et al. Prevalence of diabetic nephropathy and chronic kidney disease in diabetes mellitus in Russian Federation. Klinicheskaia nefrologiia. 2010;(3):45-50. (In Russ.)]

8. Afkarian M, Zelnick LR, Hall YN, et al. Clinical Manifestations of Kidney Disease Among US Adults With Diabetes, 1988-2014. JAMA. 2016;316(6):602610. doi: 10.1001/jama.2016.10924

9. United States Renal Data System. Annual Data Report. USRDS; 2017.

10. Бикбов Б.Т., Томилина Н.А. Состав больных и показатели качества лечения на заместительной терапии терминальной почечной недостаточности в Российской Федерации в 1998-2013 гг. // Нефрология и диализ. - 2016. - T. 18. - №2. - C. 101-164. [Bikbov BT, Tomilina NA. The contingent and treatment quality indicators in patients on replacement therapy of end stage renal disease in the Russian Federation in 1998-2013 years. Nephrology and dialysis. 2016;18(2):101-164. (In Russ.)]

11. Costacou T, Orchard TJ. Cumulative Kidney Complication Risk by 50 Years of Type 1 Diabetes: The Effects of Sex, Age, and Calendar Year at Onset. Diabetes Care. 2018;41(3):426-433. doi: 10.2337/dc17-1118

12. Gagnum V, Saeed M, Stene LC, et al. Low Incidence of End-Stage Renal Disease in Childhood-Onset Type 1 Diabetes Followed for Up to 42 Years. Diabetes Care. 2018;41(3):420-425. doi: 10.2337/dc17-0906

13. Helve J, Sund R, Arffman M, et al. Incidence of End-Stage Renal Disease in Patients With Type 1 Diabetes. Diabetes Care. 2018;41(3):434-439. doi: 10.2337/dc17-2364

14. Cho YH, Craig ME, Donaghue KC. Puberty as an accelerator for diabetes complications. Pediatr Diabetes. 2014;15(1):18-26. doi: 10.1111/pedi.12112

15. Дедов И.И., Шестакова М.В., Викулова О.К. Эпидемиология сахарного диабета в Российской Федерации: клинико- статистический анализ по данным Федерального регистра сахарного диабета // Сахарный duaбem. - 2017. - T. 20. - №1. - C. 13-41. [Dedov II, Shestakova MV, Vikulova OK. Epidemiology of diabetes mellitus in Russian Federation: clinical and statistical report according to the federal diabetes registry. Diabetes mellitus. 2017;20(1):13-41. (In Russ.)] doi: 10.14341/DM8664

16. Gargiulo R, Suhail F, Lerma EV. Cardiovascular disease and chronic kidney disease. Dis Mon. 2015;61(9):403-413. doi: 10.1016/j.disamonth.2015.07.005

\section{ИНФОРМАЦИЯ ОБ АВТОРАХ [AUTHORS INFO]}

*Викулова Ольга Константиновна, к.М.Н., доцент [Olga K. Vikulova, MD, PhD, associate professor]; адрес: Россия, 117036, Москва, улица Дм.Ульянова, д.11 [address: 11 Dm.Ulyanova street, 117036 Moscow, Russia]; ORCID: http://orcid.org/0000-0003-0571-8882; eLibrary SPIN: 9790-2665; e-mail: gos.registr@endocrincentr.ru

Шамхалова Минара Шамхаловна, А.М.н. [Minara S. Shamkhalova, MD, PhD];

ORCID: http://orcid.org/0000-0002-3433-0142; eLibrary SPIN: 4942-5481; e-mail: shamkhalova@mail.ru

Железнякова Анна Викторовна, к.м.н. [Anna V. Zheleznyakova, MD, PhD];

ORCID: http://orcid.org/0000-0002-9524-0124; eLibrary SPIN: 8102-1779; e-mail: azhelez@gmail.com

Исаков Михаил Андреевич, к.б.н. [Mikhail A. Isakov, PhD in Biology]; ORCID: http://orcid.org/0000-0001-9760-1117; eLibrary SPIN: 5870-8933; e-mail: m.isakov@aston-health.com

Шестакова Марина Владимировна, д.м.н., профессор, академик PAH [Marina V. Shestakova, MD, PhD, Professor]; ORCID: http://orcid.org/0000-0003-3893-9972; eLibrary SPIN: 7584-7015; e-mail: nephro@endocrincentr.ru

Дедов Иван Иванович, д.М.н., профессор, академик PAH [Ivan I. Dedov, MD, PhD, Professor];

ORCID: http://orcid.org/0000-0002-8175-7886; eLibrary SPIN: 5873-2280; e-mail: dedov@endocrincentr.ru

\section{ЦИТИРОВАТЬ:}

Шамхалова М.Ш., Викулова О.К., Железнякова А.В., Исаков М.А., Шестакова М.В., Дедов И.И. Эпидемиология хронической болезни почек в Российской Федерации по данным Федерального регистра взрослых пациентов с сахарным диабетом (2013-2016 гг.) // Сахарный диабет. — 2018. — Т. 21. — №3. — C. 160-169. doi: 10.14341/DM9687

\section{TO CITE THIS ARTICLE:}

Shamkhalova MS, Vikulova OK, Zheleznyakova AV, Isakov MA, Shestakova MV, Dedov II. Trends in the epidemiology of chronic kidney disease in Russian Federation according to the Federal Diabetes Register (2013-2016). Diabetes Mellitus. 2018;21(3):160-169. doi: 10.14341/DM9687 